\title{
"MY GROUP IS DISCRIMINATED AGAINST, BUT I'M NOT": DENIAL OF PERSONAL DISCRIMINATION IN FURRY, BRONY, ANIME, AND GENERAL INTEREST FAN GROUPS
}

\author{
Connor Leshner, Arizona State University \\ Dr. Stephen Reysen, Texas A \& M University at Commerce \\ Dr. Courtney N. Plante, MacEwan University \\ Daniel Chadborn, Texas A \& M University at Commerce \\ Dr. Sharon E. Roberts, Renison University College, University of Waterloo \\ Dr. Kathleen C. Gerbasi, Niagara County Community College
}

\begin{abstract}
The authors examined perceived discrimination directed toward one's fan group and toward oneself in multiple groups of fans. Specifically, furries, bronies, anime fans, and a group of miscellaneous fans completed measures assessing the extent to which they perceived discrimination toward both their fan group and toward themselves. Across all samples, participants reported greater discrimination directed toward their fan group than toward themselves, suggesting denial of personal discrimination. The difference between personal discrimination and fandom discrimination is a reliable one, as suggested by its persistence despite considerable differences between the groups with regard to the level of societal stigma. The implications of these results and future directions for this line of research are discussed.
\end{abstract}

Keywords: discrimination, stigma, furry, brony, anime, fans

\section{Introduction}

Stigmatization between groups is a long-studied phenomenon in psychology. Henry Tajfel and John Turner (1979) attributed intergroup stigmatization to a need to perpetuate a sense of positive identity by elevating one's group above others. When a group is stigmatized, it means that others are actively discriminating against them, including denying the stigmatized group access to resources. As such, stigmatized group members are often motivated to deny the discrimination put upon them. People initially belong to a group for the social affiliation and, in some cases, as a lifeline to get their needs met (Schaller \& Neuberg, 2012). When individuals experience situations where they have to decide between having access to resources and remaining with their group, then they may stay with the stigmatized group while viewing the discrimination against themselves as relatively less intense. If this effect exists in groups 
where people gain resources and fulfill their fundamental motives, such as gaining status or fulfilling a need for affiliation (Neuberg \& Schaller, 2012), then one could posit that this effect should similarly exist for fan groups, where similar needs may also be fulfilled. We present a test of this hypothesis in the present study. First, the literature on fan groups and the denial of personal discrimination against fans will be reviewed, followed by a review of the different fan groups included in the present research. We then describe the results of a large-scale study that sought to test whether denial of personal discrimination exists in fan groups as it does toward other stigmatized groups. Finally, we discuss the results and their implications for the reviewed literature.

\section{Fan Groups}

The term "fan" is derived from the term "fanatic," and refers to a person with a zealous interest in a particular topic (Phelps, 1995; Reysen, 2006). While often conceptualized as a personal identity, social scientists have increasingly studied fans in the context of fandoms, groups of like-minded people who share a similar fan-like interest (Reysen, 2006). Adapted from Tajfel's (1978) social identity theory, fandoms, like any social group, are comprised of individuals who construct social identities in line with the goals and demands of the group and in service of supporting and sustaining the group. Fandoms are a social group that, like other social groups, will develop group norms to forward the group's central values, ensure the group's survival (Feldman, 1984), create and maintain language (Eble, 1996), influence the actions for those who strongly identify with the group (Terry \& Hogg, 1996), and help to fulfill the need to belong for members of the fan group (Baumeister \& Leary 1995). Previous research has found that some fan groups, such as sport fans, are similar to other fan groups, such as media fans (Reysen \& Branscombe, 2010). Despite this, other research (Schroy, Plante, Reysen, Roberts, \& Gerbasi, 2016) has shown that fan groups also differ with regard to the underlying motivation of those comprising the group. As such, when studying fan groups, it is often necessary to test hypotheses among a variety of fan groups to determine the generalizability of the effect. Thus, examining groups parallel to each other will offer key insights into how members of groups view discrimination against their group, as well as a lesser feeling of discrimination toward the self, known as the denial of personal discrimination.

\section{Denial of Personal Discrimination}

Faye Crosby (1984) examined the cognitive and emotional barriers that may cause people to deny the existence of discrimination against the self. She investigated differences in grievances at home and at work, including differences in sex and pay discrimination. However, where she expected to find differences in grievances, she found instead that 
women, aware of discrimination, did not report grievances that affected them specifically. According to Crosby, low-status group members are motivated to deny their own victimization, rather than the much-harder-to-deny existence of discrimination against the group. This phenomenon, defined as denial of personal discrimination, refers the fact that individuals are motivated to perceive themselves as less discriminated against than the group they belong to.

In an application of Crosby's theory, researchers proposed the personal/group discrimination discrepancy, wherein people report significantly lower feelings of personal discrimination compared to their feelings of group discrimination (Crosby, 1984; Taylor, Wright, Moghaddam \& Lalonde, 1990). This effect was replicated in a later study by Dion and Kawakami (1996), where the authors found personal discrimination was always lower than reports of group discrimination for both visible (racial and gender) and invisible (culture, religion) groups. These researchers have shown the existence of self-protecting barriers that cause people to feel less discrimination, such as against their gender or race. The stigmatized person views themselves as an individual rather than a group, and this carries a number of important implications when considering fan groups.

To date, studies have examined the role of personal and group discrimination in gender (Crosby, 1984) and racial groups (Taylor et al., 1990), as well as in both groups at the same time (Dion et al., 1996). There has, however, been no examination of this theory within the context of fandoms. There is evidence to suggest discrimination between fan groups: anime fans tend to stigmatize furries (Reysen, Plante, Roberts, \& Gerbasi, 2017) and in a study assessing fantasy sports fans' feelings of furries, bronies, and anime fans, fantasy sports fans rated the other groups significantly less positively (Roberts, Plante, Reysen, \& Gerbasi, 2016). Given that fan groups often experience many of the same intraand intergroup effects as other groups, and given that fan groups can often be highly stigmatized, there is value in knowing whether these findings can be replicated in fan groups, especially given that, unlike race or sex, fan groups represent an identity that is achieved or chosen, not ascribed.

Finally, there is research assessing individuals' feelings toward fan groups: participants from an undergraduate psychology course rated a number of different fan groups, and the participants rated sport, media, and music fandoms highly in normality and prototypicality, and furries, bronies, and anime fans much lower (but anime fans were rated more highly than the other two groups; Reysen \& Shaw, 2016). Also, non-anime-fans stereotyped anime fans as socially awkward and detached from reality (Reysen, Plante, Roberts, Gerbasi, Mohebpour, \& Gamboa, 2016). Therefore, given that previous research has shown that denial of personal discrimination exists in members of stigmatized racial groups, we expect members of stigmatized fan 
groups will feel less personal discrimination relative to group discrimination. One of the most important distinctions from this research is that there is a difference in discrimination between directed toward groups, some groups are less stigmatized compared to other groups (Roberts et al., 2016), and other fan groups may not be stigmatized at all for various reasons (such as the size of the group or the association the group has with the prototypical "fan") (Reysen \& Shaw, 2016). Ultimately, there is evidence of the existence of stigma within and between different fan groups, and that some groups face more stigmatization than others.

\section{Fan Interests Sampled}

According to previous research, fantasy sport fans, a mainstream fan group, reported negative attitudes toward furries (Roberts et al., 2016). This may be due to media portrayals of furries as sexual deviants (Zuiker \& Stahl, 2003; for a formal review of furry discrimination, see Roberts et al., 2016). From such portrayals, negative stereotypes are often attached to the fandom, leading many furries to conceal their furry identity from others (Mock, Plante, Reysen, \& Gerbasi, 2013; Plante et al., 2014; Roberts et al., 2016).

In the present research we sampled furries, bronies, anime fans, and college students with varied favorite fan interests (sport, music, media, hobby). Furries are fans of media which feature anthropomorphized animal characters
(Gerbasi et al., 2008; Plante, Roberts, Reysen, \& Gerbasi, 2014), expressing this interest through, among other means, the generation of animal-themed avatars, consumption of furry-themed media, and the wearing of animalthemed mascot costumes known as "fursuits" (Plante, Reysen, Roberts, \& Gerbasi, 2016).

Bronies are adult fans of the most recent iteration of the television show My Little Pony: Friendship is Magic. Prior literature has examined some of the reasons for bronies' zealous interest in the show (for a full review see Chadborn, Plante, \& Reysen, 2016). Bronies, as a group, face a degree of stigmatization that is not unlike that experienced by furries; however, while furries have an interest in media featuring anthropomorphized animal characters, bronies take their interest from the show My Little Pony: Friendship is Magic specifically. One important distinction between furries and bronies is that, despite both subject matters centering on anthropomorphized animals, bronies do typically have less interest in costuming, although some may make their own costumes at different types of gatherings (Leshner \& De La Garza, in press).

Finally, anime fans are enthusiasts of Japanese animation, and graphic novels and comics. Similar to furries, some anime fans will dress up in a style of clothing known as cosplay (a portmanteau of costume and roleplay), wherein fans dress and act as characters from a favorite anime series (Leshner \& De La Garza, in press). Stereotypes against 
anime fans have been examined in past work, such as they lack social skills or play "too many video games" (Reysen et al., 2016; Reysen et al., 2017), and only a small proportion of stereotypes actually held true for the group. Despite this, research suggests that anime fans are not as stigmatized as either furries or bronies (Roberts et al., 2016) owing to more of its mainstream acceptance (Reysen \& Shaw, 2016).

\section{Overview of Present Research}

The purpose of the present study is to test the hypothesis that among various fan groups, including furries, bronies, anime fans, and more mainstream fans, there is a tendency for fans to deny personal discrimination while nevertheless acknowledging discrimination toward their fan group. Past research has shown that, among members of stigmatized groups, there is a tendency for members to deny personal discrimination relative to group discrimination (Crosby, 1984). This tendency has been shown among both racial and gender groups (Dion et al., 1996; Taylor et al., 1990), but to our knowledge, there is no research that has examined this effect in fan groups. The present study is a test of the generalizability of the personal/group discrimination discrepancy to groups which, unlike gender or racial groups, which are ascribed, are instead volitional or achieved. We hypothesize that the same denial of personal discrimination will exist among fan groups, in part because social identities operate within fan groups in much the same way they do within other groups (Sherif et al., 1961; Tajfel, 1978). We collected respondents' perception of discrimination toward the group and the self in groups of furries, bronies, anime fans, and a sample of college students who identified as fans of music, sports, media, and hobby-related activities. We predict that each group will report higher feelings of group discrimination than personal discrimination. As well, we predict that members of more stigmatized fan groups will report higher feelings of stigmatization, both group and personal, than members of mainstream groups.

\section{Method}

\section{Participants and Procedure}

Participants included furries $(N=$ $976,74.4 \%$ male, $0.8 \%$ other sex; $M_{\text {age }}=$ 26.39, $S D=7.10$ ) solicited at Anthrocon (furry convention in Pittsburgh, PA), bronies $(N=985,89.3 \%$ male, $1.3 \%$ other sex; $\left.M_{\text {age }}=24.47, S D=6.84\right)$ solicited from fan-related websites and message boards, anime fans $(N=737$, 54.8 male, $0.7 \%$ other sex; $M_{\text {age }}=25.01$, $S D=8.13$ ) solicited from A-Kon (an anime convention in Dallas, TX) and anime-related websites, and college students $\left(N=828,73.5 \%\right.$ female; $M_{\text {age }}=$ $21.90, S D=5.87)$ at Texas A \& M University at Commerce.

College students completed the study regarding their favorite fan interest. Their favorite fan groups were split into four groups based on prior research 
(Reysen \& Branscombe, 2010), and included: sport $(n=210)$, music $(n=222)$, media $(n=286)$, and hobby $(n=110)$. In each of the above samples, participants completed measures regarding perceived discrimination toward the group and self as part of larger surveys concerning fandom.

\section{Measures}

Participants completed two items to assess group (i.e., "Furries are discriminated against") and personal (i.e., "I have felt discriminated against because I am a furry") discrimination. Responses were made on a 7-point Likert-type scale, from $1=$ strongly disagree to $7=$ strongly agree. Each of the scales were adapted for the participants being measured (e.g., "furry" was changed to "brony" for bronies, "anime fans" for anime fans).

\section{Results}

\section{Differences in Perceived Discrimina- tion between Individuals in Groups}

To examine whether fans of different interests display denial of personal discrimination, we conducted a series of repeated measures ANOVAs with the ratings of group and self-discrimination for each of the fan categories. As shown in Table 1, furries, bronies, and anime fans all rated their group as more discriminated against than they themselves were. Furthermore, this effect occurred in sport, music, media and hobby fan groups. Thus, across both the undergraduate fan groups (sports fans, movie fans, music fans and hobby fans) and the furry, brony and anime fan groups, fans perceive they are less discriminated against than their group as a whole.

\section{Perceived Group Discrimination be- tween Fan Groups}

To examine whether fans of the different interests sampled in the present research differ with respect to perceived group discrimination, we conducted an ANOVA with group as the independent variable and perceived group discrimination as the dependent variable, $F(6$, $3519)=131.88, p<.001, \eta_{\mathrm{p}}{ }^{2}=.184$ (see Table 1 for means). Post hoc analyses showed that furry and brony fans did not differ significantly, however, furries rated group discrimination higher than the other groups. Brony and anime fans did not differ, but anime fans perceived greater group discrimination than the four college student fan interests. Sport, music, and hobby did not differ from one another, but sport and music fans perceived more group discrimination than media fans. Lastly, hobby and media fans did not differ in perceived group discrimination.

\section{Perceived Personal Discrimination between Fan Groups}

To examine differences between perceived personal discrimination between groups, we conducted an ANOVA with 
fan interest as the independent variable and perceived personal discrimination as the dependent variable, $F(6,3519)=$ 47.94, $p<.001, \eta_{\mathrm{p}}{ }^{2}=.076$ (see Table 1 for means). Post hoc analyses showed that furries rated personal discrimination significantly higher than participants in all other groups. Anime and brony fans did not differ in ratings, but rated personal discrimination higher than the four categories of fan interests for in the college student sample. Sport, music, and hobby fans did not differ, but sport was higher than media fans. Lastly, music, hobby, and media fans did not differ.

\section{Discussion}

The purpose of the present research was to test whether the denial of personal discrimination often observed within ascribed group identities like gender or race also exist within volitional, achieved identities such as fan groups. We predicted, based on prior research (e.g., Crosby, 1984; Dion et al., 1996; Taylor et al., 1990), that fans in all of the groups sampled would report greater perceived group discrimination than personal discrimination. We also predicted that less mainstream fans, such as furries, bronies, and anime fans would experience greater stigmatization in general, as has been found in prior research. The obtained results resoundingly support both hypotheses. The results showed that denial of personal discrimination is not limited to ascribed identities such as race or gender: denial of personal discrimination also exists in fan groups where identity is chosen by the individual. It is also worth noting that this denial of personal discrimination was robust, having been found across all groups. This suggests that the denial of personal discrimination exists even in groups that are not highly stigmatized. This effect, to our knowledge, has never been shown in fan groups, and helps to build a better understanding of how fan groups may feel similar group dynamics compared to other groups where identity is biological. We also found evidence that, as predicted, furries, bronies, and anime fans felt more discriminated against, both personally and as a group, than fans of more mainstream fan interests (sport, music, media, hobby).

One future direction would be to examine whether the ability to hide one's fan identity would affect their denial of personal discrimination, as well as their actions around members of outgroups. When members of stigmatized groups experience stigma, one possible response is to choose to hide their interests from peers and partners so as not to lose peer favor and social standing; if an individual were ostracized by their immediate peer group, they would likely face feelings of depression and loneliness (Leary, 1990). Baumeister and Tice (1990) refer to this concern as social anxiety. Ultimately, the more an individual perceives that others will discriminate against their fan interests, they will develop a distinct feeling of social anxiety, where they either hide their identity to save face, or risk losing the peers that are closest to them. This is further evidence about why 
individuals may put barriers up against how they view discrimination against themselves (personal discrimination) compared to how they view their group is discriminated against (group discrimination).

This research shows that discrimination happens in fan groups, and that other effects, such as denial of personal discrimination, exists in fan groups, just like it does in stigmatized racial and gender groups (Crosby, 1984; Taylor et al., 1990). This implies that people hold onto their fan identity in a way that parallels how people feel about their racial, sex or gender identity, but future research is needed to confirm these ideas. Future research is also needed to examine if stigmatized fan groups suffer from the same debilitating effects of minority status (Lord \& Saenz, 1985; Steele \& Aronson, 1995). Showing this key similarity of fan groups to minority groups would continue to lead to a better understanding to how fan groups, based on level of stigmatization, respond to discrimination. Showing that the denial of personal discrimination exists in groups where volitional identity is the unifying trait is an important step for understanding differences and similarities between groups. Future research is needed to examine these differences between groups, but this research is a necessary step for investigating the nuances that define group differences overall.

The results also give credence to why there are special events that have been created specifically to host individ- uals with like-minded interests. While future research will be necessary to confirm the accuracy of this claim, the act of having events that are entirely singlegroup oriented may create the opportunity to engage in prosocial behaviors, which may be stigmatized if performed among the general population. With the present conclusions of the research, a possible next step would be confirming how prosocial behaviors affect people when they are around other members of their group, as well as how feelings of stigmatization change before and after events.

Despite the novelty of the findings, there are limitations of the present research. First, the surveys were administered in English. Thus, fans that speak a different language were unable to participate. Additionally, most participants across the different samples were from the US. Future researchers should examine whether the same tendency to deny personal discrimination relative to group discrimination is observed in other cultures. At present, the lack of understanding of how other cultures would respond to these measures ultimately reduces the generalizability of our results. A second limitation of the present study stems from the use of a single item to assess each of the felt discrimination constructs, leading to concerns about their validity. Interpretations of questions are susceptible to the participant's own beliefs and idiosyncrasies, and single-item questionnaires may not elucidate information about why someone answered a question a certain way, only 
that they, in-fact, answered the question. A third limitation of the present study is its lack of an objective measure of stigmatization. All responses assess the extent to which individuals feel discriminated against, rather than assessing the frequency or extent to which individuals and groups themselves are actually subject to discrimination or prejudice. A final limitation of the present study is its lack of outcome variables used. For instance, participants were not surveyed on how they felt about being discriminated against. As such, the potential impact of this discrimination on well-being can only be inferred, as can many of the potential long-term implications of the results. Due to the study's correlational nature, which limits the ability to test questions of such a directional nature, future research is needed which attempts to measure such outcomes within the framework of an experimental design.

\section{Conclusion}

This paper shows that denial of personal discrimination exists in fan groups, when previous research had only shown a denial of personal discrimination in race, gender and socioeconomic status groups. In this research, the goal was to show that denial of personal discrimination exists in fan groups as it does in other stigmatized groups. We found that there were significant differences between most of the groups, and that the felt personal discrimination was significantly lower in each of the groups rela- tive to the felt group discrimination. An important takeaway from these results is that the denial of personal discrimination exists in fan groups, where previously this effect had only been shown in groups where identity was not volitional (i.e., gender and race are both traits determined by one's biology). If individuals feel similar psychological effects regardless of if identity is volitional or biological, then future research can determine how else groups differ based upon volitional identity. Fandom research is an important topic as it can shed light on how differences in volitional identity may emulate differences in biological identity, but more research is required to determine how parallel the effects are.

\section{References}

Baumeister, R. F., \& Leary, M. R. (1995). The need to belong: Desire for interpersonal attachments as a fundamental human motivation. Psychological Bulletin, 117(3), 497529. doi:10.1037/0033-

2909.117.3.497

Brewer, M. B. (1979). In-group bias in the minimal intergroup situation: A cognitive- motivational analysis.

Psychological Bulletin, 86(2), 307324.

Chadborn, D. P., Plante, C. N., \& Reysen, S. (2016). Perceived stigma, social identity, and group norms as predictors of prosocial behavior in a fandom. International Journal of Interactive Communication Systems and Technologies, 6, 35-49. 
Crosby, F. (1984). The denial of personal discrimination. American Behavioral Scientist, 27(3), 371-386. doi:10.1177/000276484027003008

Dion, K. L., \& Kawakami, K. (1996). Ethnicity and perceived discrimination in Toronto: Another look at the personal/group discrimination discrepancy. Canadian Journal of Behavioural Science/Revue Canadienne Des Sciences Du Comportement, 28(3), 203-213. doi:10.1037/0008400X.28.3.203

Eble, C. (1996). Slang \& sociability: Ingroup language among college students. Chapel Hill, NC: U North Carolina Press.

Feldman, D. C. (1984). The development and enforcement of group norms. The Academy of Management Review, 9(1), 47-53. doi: $10.2307 / 258231$

Gerbasi, K. C., Paolone, N., Higner, J., Scaletta, L. L. Bernstein, P. L., Conway, S., \& Privitera, A. (2008).

Furries from A to $\mathrm{Z}$ (anthropomorphism to zoomorphism). Society for Animals, 16, 197-222. doi:10.1163/156853008X323376

Leary, M. R. (1990). Responses to social exclusion: Social anxiety, jealousy, loneliness, depression, and low selfesteem. Journal of Social and Clinical Psychology, 9(2), 221-229. doi:10.1521/jscp.1990.9.2.221

Leshner, C. E., \& De La Garza, S. A. (in press). Dress for success: How cosplay plays a role in relationship dynamics. Journal of Interpersonal Relations, Intergroup Relations and Identity, 12(1).

Lord, C. G., \& Saenz, D. S. (1985). Memory deficits and memory surfeits: Differential cognitive consequences of tokenism for tokens and observers. Journal of Personality and Social Psychology, 49(4), 918-926. doi:10.1037/00223514.49.4.918

Markus, H. R., \& Kitayama, S. (1991). Culture and the self: Implications for cognition, emotion, and motivation. Psychological Review, 98(2), 224253. doi: $10.1037 / 0033-$ 295X.98.2.224

Mock, S. E., Plante, C. N., Reysen, S., \& Gerbasi, K. C. (2013). Deeper leisure involvement as a coping resource in a stigmatized leisure context. Leisure/Loisir, 37, 111-126.

Phelps, M.F., ed. (1995). Roget's II: The new thesaurus (3rd Ed.). Boston: Houghton Mifflin.

Plante, C. N., Roberts, S. E., Reysen, S., $\&$ Gerbasi, K. C. (2014). "One of us": Engagement with fandoms and global citizenship identification. Psychology of Popular Media Culture, 3(1), 49-64. doi:10.1037/ppm0000008

Plante, C. N., Reysen, S., Roberts, S. E., \& Gerbasi, K. C. (2016).

FurScience! A summary of five years of research from the International Anthropomorphic Research Project. Waterloo, Ontario: FurScience.

Reysen, S. (2006). Secular versus religious fans: Are they different?: 
An empirical examination. The

Journal of Religion and Popular

Culture, 12(1).

doi:10.3138/jrpc.12.1.001

Reysen, S., Lloyd, J. D., Katzarska-

Miller, I., Lemker, B. M., \& Foss, R.

L. (2010). Intragroup status and social presence in online fan groups. Computers in Human Behavior, 26(6), 1314-1317. doi:10.1016/j.chb.2010.04.003

Reysen, S., Plante, C. N., Roberts, S. E., \& Gerbasi, K. C. (2017). Accuracy of perceived prejudice toward one's fan group. The Phoenix Papers, 3(1), 122-129.

Reysen, S., Plante, C. N., Roberts, S. E., Gerbasi, K. C., Mohebpour, I., \& Gamboa, A. (2016). Pale and geeky: Prevailing stereotypes of anime fans. The Phoenix Papers, 2(1), 78-103.

Reysen, S., Plante, C. N., Roberts, S. E., Gerbasi, K. C., \& Shaw, J. (2016). An examination of anime fan stereotypes. The Phoenix Papers, 2(2), 90-117.

Reysen, S., \& Shaw, J. (2016). Sport fan as the default fan: Why non-sport fans are stigmatized. The Phoenix Papers, 2(2), 234-252.

Reysen, S., Plante, C., \& Chadborn, D. (2017). Better together: Social connections mediate the relationship between fandom and well-being. AASCIT Journal of Health, 4, 68-73.

Roberts, S. E., Plante, C. N., Gerbasi, K. C., \& Reysen, S. (2015). The anthrozoomorphic identity: Furry fandom members' connections to nonhuman animals. Anthrozoös,
28(4), 533-548.

doi:10.1080/08927936.2015.106999 3

Roberts, S. E., Plante, C. N., Reysen, S., \& Gerbasi, K. C. (2016). Not all fantasies are created equal: Fantasy sport fans' perceptions of furry, brony, and anime fans. The Phoenix Papers, 2(1), 40-60.

Schaller, M. \& Neuberg, S. L. (2012). Danger, disease, and the nature of prejudice(s). Advances in Experimental Social Psychology, 46, 1-54. doi:10.1016/B978-0-12394281-4.00001-5

Schroy, C., Plante, C. N., Reysen, S., Roberts, S. E., \& Gerbasi, K. C. (2016). Different motivations as predictors of psychological connection to fan interest and fan groups in anime, furry, and fantasy sport fandoms. The Phoenix Papers, 2(2), 148-167.

Sherif, M., Harvey, O. J., White, B. J., Hood, W. R., \& Sherif, C. W. (1961). Intergroup conflict and cooperation: The Robbers Cave experiment (Vol. 10). Norman, OK: University Book Exchange.

Steele, C. M., \& Aronson, J. (1995).

Stereotype threat and the intellectual test performance of African Americans. Journal of Personality and Social Psychology, 69(5), 797811. doi:10.1037/00223514.69.5.797

Tajfel, H. (1978). Social categorization, social identity and social comparison. In H. Tajfel (Ed.), Differentiation between social 
groups (pp. 61-76). London:

Academic Press.

Tajfel, H., \& Turner, J. (2001). An integrative theory of intergroup conflict. In M. A. Hogg, \& D. Abrams (Eds.), Intergroup relations: Essential readings (pp. 94-109). New York, NY: Psychology Press. Taylor, D. M., Wright, S. C., Moghaddam, F. M., \& Lalonde, R. N. (1990). The personal/group discrimination discrepancy: Perceiving my group, but not myself, to be a target for discrimination. Personality and Social Psychology Bulletin, 16(2), 254-262. doi:10.1177/0146167290162006

Terry, D. J., \& Hogg, M. A. (1996).

Group norms and the attitude- behavior relationship: A role for group identification. Personality and Social Psychology Bulletin, 22(8), 776-793. doi:10.1177/0146167296228002 Zuiker, A. E. (Writer), Stahl, J. (Writer), \& Lewis, R. (Director). (2003). Fur and loathing [Television series episode]. In J. Bruckheimer (Producer), CSI: Crime Scene Investigation. Los Angeles, CA: CBS Paramount Network Television. Address correspondence to Stephen Reysen, Department of Psychology, Texas A \& M University at Commerce, Commerce, Texas, 75429. E-mail: Stephen.Reysen@tamuc.edu 
Table 1

Repeated Measures ANOVA of Perceived Group and Personal Discrimination Across Studies

\begin{tabular}{|c|c|c|c|c|c|c|}
\hline Sample & $\begin{array}{l}\text { Group } \\
\text { Mean }(S D)\end{array}$ & $\begin{array}{l}\text { Personal } \\
\text { Mean }(S D)\end{array}$ & $\begin{array}{l}d f \\
\text { error }\end{array}$ & $F$-Value & $p$-Value & $\eta_{\mathrm{p}}^{2}$ \\
\hline Furry & $4.64(1.64)_{\mathrm{a}}$ & $3.75(2.00)_{\mathrm{a}}$ & 975 & 279.79 & $<.001$ & .22 \\
\hline Brony & $4.43(1.64)_{\mathrm{ab}}$ & $3.19(1.87)_{b}$ & 984 & 604.86 & $<.001$ & .38 \\
\hline Anime & $4.09(1.87)_{\mathrm{b}}$ & $3.14(2.07)_{b}$ & 736 & 274.76 & $<.001$ & .27 \\
\hline \multicolumn{7}{|c|}{ College Student Sample } \\
\hline \multicolumn{2}{|c|}{ Sport $2.83(1.71)_{\mathrm{c}}$} & $1.74)_{\mathrm{c}}$ & 11.49 & .001 & .05 & \\
\hline Music & $2.80(1.73)_{\mathrm{c}}$ & $2.37(1.63)_{\mathrm{c}}$ & 221 & 27.09 & $<.001$ & .11 \\
\hline Media & $2.27(1.53)_{\mathrm{d}}$ & $2.02(1.50)_{\mathrm{d}}$ & 285 & 15.71 & $<.001$ & .05 \\
\hline Hobby & $2.64(1.53)_{\mathrm{cd}}$ & $2.22(1.48)_{\mathrm{cd}}$ & 109 & 15.11 & $<.001$ & .12 \\
\hline
\end{tabular}

Note. Responses made on a 7 -point response scale, from $1=$ strongly disagree to $7=$ strongly agree. Subscripts that differ indicate significant $(p<.05)$ difference between means within each column. 